\title{
Automated Atom-by-Atom Assembly of Structures in Graphene: The Rise of STEM for Atomic Scale Control
}

\author{
Ondrej Dyck, ${ }^{1,2}$ Songkil Kim, ${ }^{3}$ Andrew R. Lupini, ${ }^{2,4}$ Sergei V. Kalinin ${ }^{1,2}$ and Stephen Jesse ${ }^{1,2}$ \\ ${ }^{1 .}$ Center for Nanophase Materials Science, Oak Ridge National Laboratory, Oak Ridge, USA. \\ 2. Institute for Functional Imaging of Materials, Oak Ridge National Laboratory, Oak Ridge, USA. \\ 3. Department of Mechanical Engineering, Pusan National University, Republic of Korea. \\ 4. Materials Science and Technology Division, Oak Ridge National Laboratory, Oak Ridge, USA.
}

Since the successful manipulation of single atoms by Don Eigler[1] in the late 80ies and early 90ies using a scanning tunneling microscope (STM) captivated the scientific community by the possibility of building materials and devices from the atom up. For over twenty years the STM was the only instrument capable of single atom manipulation. In recent years, however, the scanning transmission electron microscopy (STEM) community has made significant strides in developing the capabilities of STEM for this purpose.[2] In this manuscript, we outline the development of an atom manipulation platform which addresses various challenges with the current modes of manipulation through automated feedback, custom beam control, and real time data processing.

Previous demonstrations of single dopant movement through a graphene lattice[3, 4] have relied on sequential imaging and manual positioning of the electron beam. This process is slow and tedious and prevents imaging of the sample during the manipulation stage, requiring continual reimaging to check the sample state which exposes the lattice to additional irradiation which can lead to unintended alterations. Here, we mitigate these problems through a custom beam control and feedback platform which allows real time imaging during atomic manipulation, automated lattice recognition, and automated beam positioning for the movement of dopant atoms through a graphene lattice.

Figure 1 a) shows the software controlling the beam of a UltraSTEM U100 STEM accessed through a custom hardware interface to control the microscope scan coils and collect and process detector output. During the manipulation stage the beam is scanned in a spiral around the desired lattice site. The red, dotted inset shows a false colormap of the beam fluence across the irradiated area. Due to the shape of the spiral, the vast majority of the irradiation occurs at the center of the irradiated area, allowing a concentrated dose at one position while simultaneously enabling a larger image of the surrounding area to be viewed. The black, dotted inset highlights the image generated during the manipulation stage where a bright Si dopant can be clearly seen. The majority of the beam fluence, however, is incident upon the neighboring $\mathrm{C}$ atom. When the carbon atom is almost ejected from the lattice a bond reversal occurs and the $\mathrm{C}$ and $\mathrm{Si}$ switch places in the lattice.[5] The black, dotted inset highlights the observed state just after the transition has occurred with the arrow indicating the movement of the Si dopant.

Figure $1 \mathrm{~b}$ ) illustrates the results of a decision-making routine which decides how to navigate through a hexagonal lattice to automatically decide where to position the beam at each step to move an atom from point A to point $\mathrm{B}$. The green line represents a direct path and the blue dots represent the path chosen by the routine. Figure $1 \mathrm{c}$ )-e) illustrate an improved atom detection scheme. The raw data acquired through a sparse, noisy scan is shown in c). The power spectrum (FFT) of the raw data is analyzed to locate the strongest periodicities and ring filtered (indicated by the overlaid doughnut) to produce the cleaned image shown in e). This cleaned image is cross correlated with a synthetic lattice image generated based 
on the position of the peaks in the FFT to estimate the positions of each atom in the image. This procedure makes an assumption that the image is of a perfect single crystal (a constraint) but enables reliable detection of atomic positions from extremely noisy (i.e. rapidly acquired) data.

Finally, in f)-h), we illustrate e-beam induced movement of a single Si substitutional dopant atom a distance of about $3 \mathrm{~nm}$ through the graphene lattice. The images were artificially colored using the fire look up table in ImageJ. The black line in $g$ ) and $h$ ) shows a representative path through the lattice for illustration (not the actual path taken).

These developments represent a significant step toward converting the STEM from a characterization platform to a full-fledged atom-by-atom manipulation platform.

References:

[1] Eigler, D.M. and E.K. Schweizer, Nature 344 (1990), p. 524.

[2] Kalinin, S.V. and S.J. Pennycook, MRS Bulletin 42 (2017), p. 637.

[3] Susi, T. et al, 2D Materials 4 (2017), p. 042004.

[4] Dyck, O. et al, Applied Physics Letters 111 (2017), p. 113104.

[5] Susi, T. et al, Physical Review Letters 113 (2014), p. 115501.

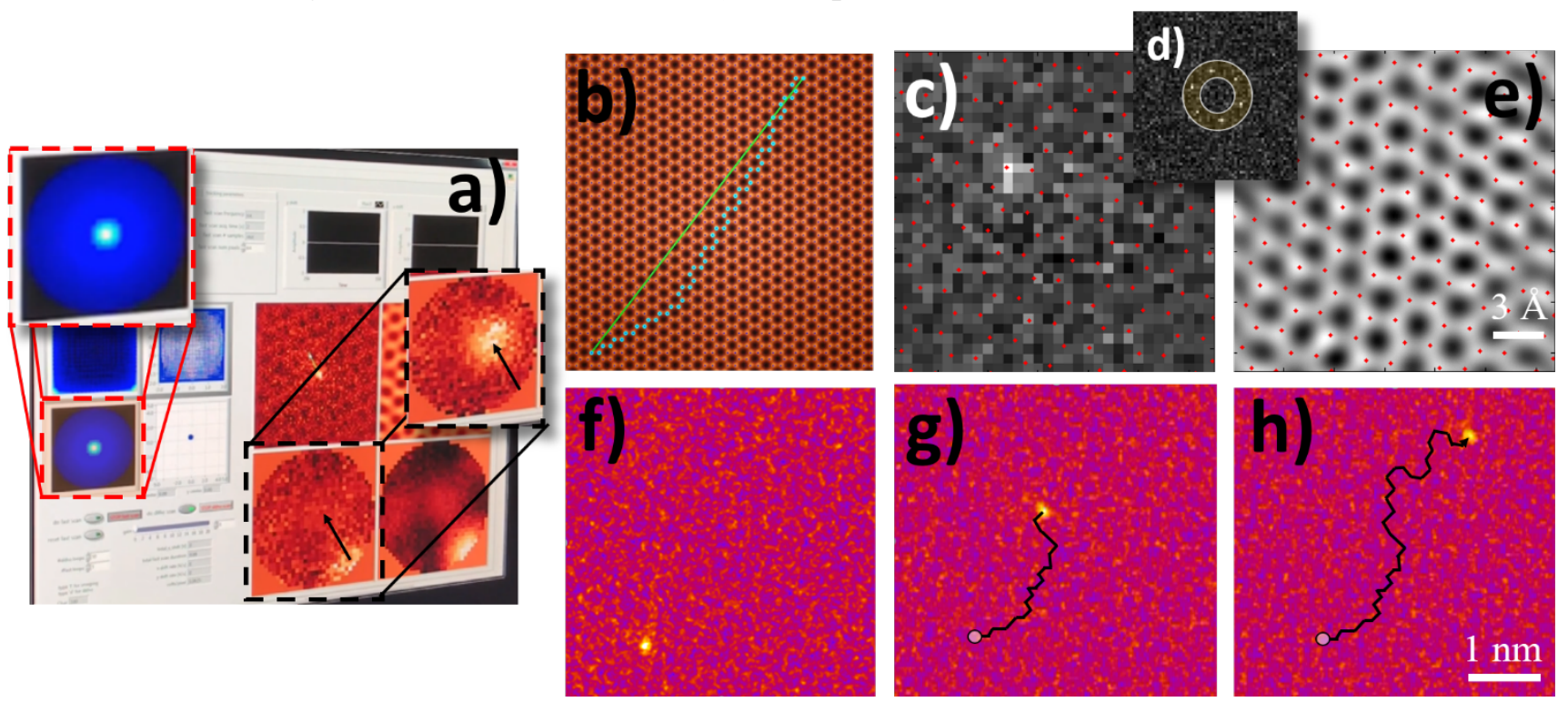

Figure 1: Summary of the real-time image analysis and beam control interface. a) shows a picture of the software in use with the red, dotted inset highlighting illustrating the beam fluence localized in the center of the spiral scan. The black, dotted inset shows the real-time imaging during the manipulation step with the black arrow indicating the movement of the bright Si dopant atom. b) illustrates the results of a decisionmaking routine that plans a viable route through a lattice. The green line represents the shortest Euclidean distance from point A to point $\mathrm{B}$, and the blue dots represent the computer's planned pathway through the lattice. c)-e) demonstrate the atom finding capabilities. c) shows the raw data from a sparsely scanned image. d) shows the FFT of c) where a ring filter is applied to the detected peaks, illustrated by the overlaid doughnut. e) shows the filtered result. e) is cross correlated with an artificial lattice image generated from the peaks in the FFT (not shown) to determine the atom positions which are illustrated by the red dots. f)-h) show the motion of a single Si substitutional defect through the graphene lattice. The black line is an illustrated path and does not represent the actual path of motion. 